\title{
On sets of natural numbers without solution to a noninvariant linear equation
}

\author{
by
}

Tomasz Schoen (Kiel*)

Let us consider a linear equation (*)

$$
a_{1} x_{1}+\ldots+a_{k} x_{k}=b,
$$

where $a_{1}, \ldots, a_{k}, b \in \mathbb{Z}$. We call the equation $(*)$ invariant if both $s=$ $a_{1}+\ldots+a_{k}=0$ and $b=0$, and noninvariant otherwise. We say that a set $A$ is $(*)$-free if it contains no nontrivial solution to $(*)$ and define $r(n)$ as the size of the largest $(*)$-free set contained in $[n]=\{1, \ldots, n\}$.

The behavior of $r(n)$ has been extensively studied for many cases of invariant linear equations. The two best known examples are the equation $x+y=2 z$, when $r(n)$ is the size of the largest set without arithmetic progression of length three contained in $[n]$ (see [6]), and the equation $x_{1}+$ $x_{2}=y_{1}+y_{2}$, when $r(n)$ becomes the size of the largest Sidon subset of $[n]$ (see [3], [7], [8]).

Much less is known about the behavior of $r(n)$ for noninvariant linear equations, maybe apart from sum-free sets (see for example [1], [2], [5], [10]). The main contribution to this subject was made by Ruzsa [9] who studied properties of sets without solutions to a fixed noninvariant linear equation. Following his paper let us define

$$
\begin{aligned}
& \bar{\Lambda}(*)=\sup \{\overline{\mathrm{d}}(A): A \subseteq \mathbb{N}, A \text { is }(*) \text {-free }\}, \\
& \underline{\Lambda}(*)=\sup \{\underline{\mathrm{d}}(A): A \subseteq \mathbb{N}, A \text { is }(*) \text {-free }\}, \\
& \bar{\lambda}(*)=\limsup _{n \rightarrow \infty} r(n) / n, \\
& \underline{\lambda}(*)=\liminf _{n \rightarrow \infty} r(n) / n,
\end{aligned}
$$

where $\overline{\mathrm{d}}(A), \underline{\mathrm{d}}(A)$ denote the upper and lower density of the set $A$. Sometimes, we write just $\bar{\Lambda}, \underline{\Lambda}, \bar{\lambda}, \underline{\lambda}$ instead of $\bar{\Lambda}(*), \underline{\Lambda}(*), \bar{\lambda}(*), \underline{\lambda}(*)$.

2000 Mathematics Subject Classification: 11B75, 11A99.

*On leave from Adam Mickiewicz University, Poznań, Poland. 
The aim of this paper is to answer the following questions posed in Ruzsa's paper [9].

1. Does there exist an absolute constant $C$ such that for every noninvariant linear equation we have

$$
C \bar{\Lambda} \geq \underline{\lambda} ?
$$

2. Let $\varepsilon>0$ be an arbitrary number. Is it possible to find a noninvariant equation with $s \neq 0$ and $\bar{\lambda}<\varepsilon$ ?

3 . Is it true that for every noninvariant linear equation we have

$$
\Lambda=\bar{\Lambda}=\underline{\Lambda} ?
$$

4. For an integer $m>1$, let $\varrho(m)$ denote the maximal cardinality of a (*)-free set $A \subseteq \mathbb{Z}_{m}$. Put

$$
\varrho=\sup \varrho(m) / m \text {. }
$$

Is it true that always

$$
\bar{\lambda}=\underline{\lambda}=\max \left(\varrho, \frac{s^{+}-s^{-}}{s^{+}}\right),
$$

where $s^{+}=\sum_{a_{i}>0} a_{i}, s^{-}=\sum_{a_{i}<0} a_{i}$ (we may assume that $s^{+}>0$ and $\left.s^{+} \geq s^{-}\right) ?$

Notation. In this note $[n]=\{1, \ldots, n\}$ and $[u, w]=\{u \leq n \leq w: n \in$ $\mathbb{N}\}$. We also set $A k=\{a k: a \in A\}$ and $h A=\left\{a_{1}+\ldots+a_{h}: a_{1}, \ldots, a_{h} \in A\right\}$. We use $\operatorname{gcd}\{A\}$ to denote the greatest common divisor of the elements of the set $A$, and set $s \pm A=\{s \pm a: a \in A\}$. Finally, $A(n)$ denotes the counting function of $A$, i.e. $A(n)=|A \cap[n]|$.

In order to deal with the first question we use the following result of Euczak and Schoen [5].

Theorem A. If $A \subseteq \mathbb{N}$ and there is no solution to the equation $y=$ $x_{1}+\ldots+x_{k}$, then

$$
\overline{\mathrm{d}}(A) \leq 1 / \rho(k-1),
$$

where $\rho(k)=\min \{m \in \mathbb{N}: m$ does not divide $k\}$.

Now we can answer the first from Ruzsa's questions in the negative.

THEOREM 1. There is no an absolute constant $C$ such that

$$
C \bar{\Lambda} \geq \underline{\lambda}
$$

for every linear equation. Moreover, for every $\varepsilon>0$ there is an equation such that $\bar{\Lambda}<\varepsilon$ and $\underline{\lambda}>1-\varepsilon$.

Proof. It is enough to prove that there exists a sequence of equations $\left(e_{1}\right),\left(e_{2}\right), \ldots$ such that

$$
\underline{\lambda}\left(e_{n}\right) \rightarrow 1 \quad \text { and } \quad \bar{\Lambda}\left(e_{n}\right) \rightarrow 0 \quad \text { as } n \rightarrow \infty .
$$


For a natural number $n$ set $k_{n}=n !+1$. Then, for every $n$, we have $\rho\left(k_{n}\right)>n$. Furthermore, denote by $\left(e_{n}\right)$ the equation

$$
y=x_{1}+\ldots+x_{k_{n}} .
$$

Thus, it follows from Theorem A that for every $n \in \mathbb{N}$,

$$
\bar{\Lambda}\left(e_{n}\right) \leq 1 / \rho\left(k_{n}\right)<1 / n,
$$

and so $\bar{\Lambda}\left(e_{n}\right) \rightarrow 0$ as $n \rightarrow \infty$.

On the other hand, for every $m \in \mathbb{N}$ the set $\left\{\left\lceil m / k_{n}\right\rceil+1, \ldots, m\right\}$ contains no solutions to the equation $\left(e_{n}\right)$, so

$$
\underline{\lambda}\left(e_{n}\right) \geq\left(k_{n}-1\right) / k_{n} .
$$

Consequently, $\underline{\lambda}\left(e_{n}\right) \rightarrow 1$ as $n \rightarrow \infty$, which completes the proof of Theorem 1.

In order to solve the second problem we make use of the following theorem of Lev [4].

Theorem B. Assume that $A \subseteq[n]$ and

$$
|A| \geq \frac{n-1}{k}+2 \text {. }
$$

Then there are integers $d \leq k-1, h \leq 2 k-1$ and $m$ such that

$$
\{m d,(m+1) d, \ldots,(m+n-1) d\} \subseteq h A .
$$

Furthermore, $d=\operatorname{gcd}\{A-\min A\}$ and $h$ can be chosen to be the largest multiple of $d$ less than or equal to $2 k-1$.

Ruzsa [9] showed that $\bar{\lambda}$ may not be bounded from below by a positive absolute constant. For every $\varepsilon>0$ he gave an example of a noninvariant linear equation with $s=0$ and $\bar{\lambda}<\varepsilon$ and asked: Is it possible that $s \neq 0$ ? We prove a more general result, which for a suitable choice of $k$ and $l$ provides an example of a noninvariant equation with $s \neq 0$ and arbitrarily small $\bar{\lambda}$.

Theorem 2. Suppose that $k, l \in \mathbb{N}$ and $k>l$. If $A \subseteq\{1, \ldots, n\}$ contains no solution to the equation $x_{1}+\ldots+x_{k}=y_{1}+\ldots+y_{l}$, then

$$
|A| \leq \max \left(\frac{2(k-l) n}{l},\left\lceil\frac{n}{\rho(k-l)}\right\rceil\right) .
$$

Pr o of. Suppose that the assertion does not hold, so in particular $|A|>$ $2(k-l) n / l$. Obviously, we can assume $2(k-l) / l<1$. Thus, it follows from Theorem B that there exists $a \in \mathbb{N}$ such that

$$
\{a, a+d, \ldots, a+(n-1) d\} \subseteq\lfloor l /(k-l)\rfloor A,
$$

where $d=\operatorname{gcd}\{A-\min A\}$. Furthermore, for some $b \in \mathbb{N}$ we have

$$
\{b, b+d, \ldots, b+(k-l)(n-1) d\} \subseteq l A .
$$


Note that, since $|A|>\lceil n / \rho(k-l)\rceil$, we must have $d<\rho(k-l)$, and so $k-l \equiv 0(\bmod d)$ by the definition of the function $\rho$.

Let $x \in A$ be an arbitrary number with $x<n$. Then

$$
x(k-l) \leq(k-l)(n-1) d .
$$

Hence

$$
b+x(k-l) \in\{b, b+d, \ldots, b+(k-l)(n-1) d\} \subseteq l A .
$$

Thus, there exist $x_{1}, \ldots, x_{l}, y_{1}, \ldots, y_{l} \in A$ such that

$$
b=x_{1}+\ldots+x_{l} \quad \text { and } \quad b+x(k-l)=y_{1}+\ldots+y_{l} .
$$

Hence, we arrive at

$$
x_{1}+\ldots+x_{l}+x(k-l)=y_{1}+\ldots+y_{l},
$$

which is a contradiction.

For any fixed $t \in \mathbb{N}$, set $k=(2 t+3) t$ ! and $l=(2 t+2) t$ !, which implies $\rho(k-l)>t$. Thus, Theorem 2 gives $\bar{\lambda}<1 / t$ for the equation $x_{1}+\ldots+x_{k}=$ $y_{1}+\ldots+y_{l}$.

Finally, we show that for the equation $x_{1}+x_{2}=k y$, where $k \geq 10$, neither $\Lambda=\bar{\Lambda}=\underline{\Lambda}$, nor $\underline{\lambda}=\max \left(\varrho \frac{s^{+}-s^{-}}{s^{+}}\right)$, which answers the third and the fourth question of Ruzsa. As a matter of fact, we prove that one can have $\bar{\Lambda}<\underline{\Lambda}<\underline{\lambda}$.

Let us make first the following elementary observation.

FACT. Let $A$ be a set of positive integers with no solution to the equation $x_{1}+x_{2}=k y$, where $k$ is fixed positive integer. Then $\underline{\Lambda} \leq 1 / 2$.

Pr o of. Every set $A \in \mathbb{N}$ with $\underline{\mathrm{d}}(A)>1 / 2$ contains in its sum-set $A+A$ each natural number from some point on. Thus, the sets $A+A$ and $A k$ may not be disjoint.

EXAMPle 1. For a given $k>2$ define

$$
S=\left(\bigcup_{n=0}^{\infty}\left[\frac{k^{2 n}}{2^{n}}+1, \frac{k^{2 n+1}}{2^{n+1}}\right]\right) \cap \mathbb{N} .
$$

It is clear that there is no solution to the equation $x_{1}+x_{2}=k y$ in the set $S$ and $\overline{\mathrm{d}}(S)=k(k-2) /\left(k^{2}-2\right)$, so $\bar{\Lambda} \geq k(k-2) /\left(k^{2}-2\right)$. The next theorem shows that, in fact, $\bar{\Lambda}=k(k-2) /\left(k^{2}-2\right)$.

TheOREM 3. If $A \subseteq \mathbb{N}$ contains no solutions to the equation $x_{1}+x_{2}=k y$, where $k \geq 10$, then

$$
\overline{\mathrm{d}}(A) \leq \frac{k(k-2)}{k^{2}-2} .
$$


Proof. Assume $\overline{\mathrm{d}}=\overline{\mathrm{d}}(A) \geq k(k-2) /\left(k^{2}-2\right)$. For a given $\varepsilon$ with $1 / k^{3}>\varepsilon>0$ choose $n_{\varepsilon}$ so that $A(i)<(\overline{\mathrm{d}}+\varepsilon) i$ for every $i>n_{\varepsilon}$. Let $n$ be such that $n>k n_{\varepsilon}$ and $(\overline{\mathrm{d}}-\varepsilon) n<A(n)$. Furthermore set $m=\min A$.

First, assume

$$
A \cap\left[\frac{4 n}{k^{2}-2}, \frac{2\left(k^{2}-2 k-2\right) n}{k\left(k^{2}-2\right)}\right] \neq \emptyset .
$$

For each $y_{0} \in A \cap\left[4 n /\left(k^{2}-2\right), n / k\right]$ and $x<k y_{0}$, either $x \notin A$ or $k y_{0}-x \notin A$, so

$$
A(n) \leq \frac{k y_{0}}{2}+\left(n-k y_{0}\right) \leq \frac{k^{2}-2 k-2}{k^{2}-2} n<(\overline{\mathrm{d}}-\varepsilon) n,
$$

which contradicts the choice of $n$. The case

$$
A \cap\left[\frac{n}{k}, \frac{2\left(k^{2}-2 k-2\right) n}{k\left(k^{2}-2\right)}\right] \neq \emptyset
$$

can be dealt with in a similar way.

Now suppose

$$
A \cap\left[\frac{4 n}{k^{2}-2}, \frac{2\left(k^{2}-2 k-2\right) n}{k\left(k^{2}-2\right)}\right]=\emptyset .
$$

Set

$$
\begin{aligned}
& A_{1}=A \cap\left[\frac{2 n}{k^{2}}+\frac{m}{k}, \frac{4 n}{k\left(k^{2}-2\right)}\right), \\
& A_{2}=A \cap\left(\frac{2\left(k^{2}-2 k-2\right) n}{k\left(k^{2}-2\right)}, \frac{2 n}{k}\right],
\end{aligned}
$$

and assume that neither of these sets is empty, otherwise the proof follows the same lines. Observe $\left(A_{1} k-m\right) \cap A=\emptyset$ and $\left(A_{1} k-m\right) \subseteq[2 n / k, n]$. Since $A$ has no solutions to the equation $x_{1}+x_{2}=k y$ we get

$$
|A \cap[k s-n, n]| \leq n-k s / 2,
$$

where $s=\min A_{2}$. Moreover, since $k \geq 10$, we have $k \max A_{1} \leq k s-n$. These yield

$$
|A \cap[2 n / k, n]| \leq n-2 n / k-\left|A_{1}\right|-n+k s / 2,
$$

so that

$$
\begin{aligned}
A(n) & \leq(\overline{\mathrm{d}}+\varepsilon) 2 n / k^{2}+\left|A_{1}\right|+\left|A_{2}\right|+k s / 2-2 n / k-\left|A_{1}\right|+O(1) \\
& \leq(\overline{\mathrm{d}}+\varepsilon) 2 n / k^{2}+n-2 n / k+O(1) .
\end{aligned}
$$

Thus,

$$
(\overline{\mathrm{d}}-\varepsilon) n \leq A(n) \leq(\overline{\mathrm{d}}+\varepsilon) 2 n / k^{2}+n-2 n / k+O(1),
$$

which gives

$$
\overline{\mathrm{d}} \leq \frac{k(k-2)}{k^{2}-2}
$$


EXAMPLE 2. Let $n \in \mathbb{N}$ and set

$$
\begin{aligned}
T= & \left(\left[\frac{8 n}{k\left(k^{4}-2 k^{2}-4\right)}+1, \frac{4 n}{k^{4}-2 k^{2}-4}\right]\right. \\
& \left.\cup\left[\frac{4\left(k^{2}-2\right) n}{k\left(k^{4}-2 k^{2}-4\right)}+1, \frac{2\left(k^{2}-2\right) n}{k^{4}-2 k^{2}-4}\right] \cup\left[\frac{2 n}{k}+1, n\right]\right) \cap \mathbb{N} .
\end{aligned}
$$

It is not difficult to see that $x_{1}+x_{2}=k y$ with $x_{1}, x_{2}, y \in T$ is not possible. Moreover

$$
|T|=\left(\frac{k(k-2)}{k^{2}-2}+\frac{8(k-2)}{k\left(k^{2}-2\right)\left(k^{4}-2 k^{2}-4\right)}\right) n+O(1),
$$

so

$$
\underline{\lambda} \geq \frac{k(k-2)}{k^{2}-2}+\frac{8(k-2)}{k\left(k^{2}-2\right)\left(k^{4}-2 k^{2}-4\right)} .
$$

(In fact, it is shown in [11] that the lower bound above is the actual value of $\underline{\lambda}$ for the equation $x_{1}+x_{2}=k y$.)

Since $s^{+}=k$ and $s^{-}=2$ we have $\left(s^{+}-s^{-}\right) / s^{+}=1-2 / k$. On the other hand, using the same argument as in the proof of the Fact one can show that for every set $A \subseteq \mathbb{Z}_{m}$ with no solutions to the equation $x_{1}+x_{2}=k y$, we have $|A| \leq m / 2$, thus $\varrho \leq 1 / 2$. Finally, we obtain

$$
\underline{\lambda} \geq \frac{k(k-2)}{k^{2}-2}+\frac{8(k-2)}{k\left(k^{2}-2\right)\left(k^{4}-2 k^{2}-4\right)}>1-\frac{2}{k}=\max \left(\varrho, \frac{s^{+}-s^{-}}{s^{+}}\right) .
$$

\section{References}

[1] N. Alon, Independent sets in regular graphs and sum-free sets of finite groups, Israel J. Math. 73 (1991), 247-256.

[2] J.-M. Deshoulliers, G. Freimen, V. Sós and M. Temkin, On the structure of sum-free sets, 2, Astérisque 258 (1999), 149-161.

[3] P. Erdős, V. Sós and A. Sárközy, On sum sets of Sidon sets, J. Number Theory 47 (1993), 329-347.

[4] V. Lev, Optimal representation by sumsets and subset sums, ibid. 62 (1997), 127143.

[5] T. Euczak and T. Schoen, On the infinite sum-free sets of natural numbers, ibid. 66 (1997), 211-224.

[6] K. F. Roth, On certain sets of integers, J. London Math. Soc. 28 (1953), 104-109.

[7] I. Z. Ruzsa, On infinite Sidon sequences, J. Number Theory 68 (1998), 63-71.

[8] - Solving a linear equation in a set of integers I, Acta Arith. 65 (1993), 259-282.

[9] -, Solving a linear equation in a set of integers II, ibid. 72 (1995), 385-397. 
[10] T. Schoen, On the density of universal sum-free sets, Combin. Probab. Comput. 8 (1999), 277-280.

[11] - Subsets of $\{1, \ldots, n\}$ with no solutions to the equation $x+y=k z$, in preparation.

Mathematisches Seminar

Universität zu Kiel

Ludewig-Meyn-Str. 4

24098 Kiel, Germany

E-mail: tos@numerik.uni-kiel.de

Received on 18.6.1999

and in revised form on 6.12.1999 